\title{
Does COVID-19 Affect Periodontal and Peri-Implant Diseases?
}

\author{
Mahdi Kadkhodazadeh, ${ }^{a, b}$ Reza Amid, , ${ }^{a, b}$ \& Anahita Moscowchi ${ }^{b, *}$ \\ aDentofacial Deformities Research Center, Research Institute for Dental Sciences, Shahid Beheshti University of \\ Medical Sciences, Tehran, Iran; 'Department of Periodontics, School of Dentistry, Shahid Beheshti University of \\ Medical Sciences, Tehran, Iran \\ *Address all correspondence to: Anahita Moscowchi, Department of Periodontics, School of Dentistry, Shahid Beheshti University of \\ Medical Sciences, Daneshjoo Blvd., Evin, Shahid Chamran Highway, Tehran, Iran, P.O. Box: 1983963113; Tel.: +98(21)-299-02314; \\ Fax: +98 (21)-224-39784, E-mail: a.moscowchi@gmail.com; ORCID: https://orcid.org/0000-0003-1327-5014
}

\begin{abstract}
COVID-19 has imposed tremendous burden on the healthcare systems and healthcare professionals worldwide. As periodontitis and peri-implantitis are chronic diseases, regular recall visits play a critical role in management of these conditions; however, the current situation may stop patients from attending their regular maintenance. Therefore, it may be necessary to reconsider and revise the treatment protocols.
\end{abstract}

KEY WORDS: periodontitis, peri-implantitis, dental implant, COVID-19

\section{INTRODUCTION}

The novel coronavirus disease is a respiratory infectious condition caused by severe acute respiratory syndrome coronavirus 2 (SARS-CoV-2), transmitted by human-to-human contact. ${ }^{1}$ Coronavirus disease was declared a global pandemic by the World Health Organization on March 11, 2020, imposing a heavy burden on healthcare systems and professionals worldwide. The outbreak has had profound political, economical, and emotional effects. It has also forced educational systems including dental schools to defer face-to-face training and instead provide virtual learning environments.

Periodontitis, the most common periodontal disease, has a multifactorial etiology. Bacterial pathogens have a fundamental role in development of periodontitis. ${ }^{2}$ A similar condition, referred to as "peri-implantitis," may influence peri-implant tissues. According to Slots, some viruses have been isolated from periodontal pockets. ${ }^{3}$ Thus, periodontal pockets may serve as reservoirs for pathogens. ${ }^{3}$ Considering the chronic nature of periodontitis and peri-implantitis, regular recall visits are important for managing these conditions. ${ }^{4}$ However, the current worrisome crisis may stop patients from attending their regular maintenance appointments, predisposing them to adverse consequences. In addition, the current pandemic is stressful for everyone, and psychological stress can contribute to periodontal and peri-implant attachment loss. ${ }^{5,6}$

It should also be noted that although COVID-19 is a recently identified disease, the new coronavirus was identified in the saliva of infected patients. ${ }^{7}$ Therefore, serious concerns are present regarding its transmission through contact with droplets generated during dental procedures; thus, extreme protective measures are mandatory for dental practitioners. Considering existing concerns, many dentists have chosen to close their practices, creating problems for patients seeking dental care. It may become necessary to reconsider and revise treatment protocols, because we may have this problem for a long period of time. ${ }^{8}$ As a result, several measures have been recommended:

- Inform patients about the possibility of oral complications related to the virus, because its relationship with oral vesiculobullous lesions has been reported. ${ }^{9}$

- Reinforce proper oral hygiene, providing patient education through virtual education and mass media and placing emphasis on the significance of regular maintenance visits. $^{10}$

- Encourage patients to use hydrogen peroxide mouthwash before therapeutic dental 
procedures, because the virus is highly sensitive to oxidation. ${ }^{11,12}$

- Exert caution in performing procedures that generate high amounts of droplets and aerosols, such as ultrasonic scaling and root planning. ${ }^{13}$

- Consider more conservative approaches such as crestal sinus floor elevation and placement of short implants instead of lateral window or advanced bone augmentations.

- Consider adjunctive modalities such as laser treatment and photodynamic therapy to improve periodontal health.

- Strictly adhere to infection-control protocols and personal protective measures for patients and office staff.

- Design advanced virtual-training programs to improve dental student skill in such situations.

Although emergency dental treatments must be the only interventions provided during the acute phase of the COVID-19 pandemic, conservative treatment plans based on available evidence must also be considered as priority after this crisis.

\section{CONCLUSIONS}

It seems evident that COVID-19 can affect periodontal and peri-implant diseases due to the disease's viral nature, level of stress created in communities, impairing maintenance phases due to quarantine and social-distancing programs, and the inevitable change in provision of dental care. However, further clinical studies are warranted on this topic.

\section{REFERENCES}

1. Cascella M, Rajnik M, Cuomo A, Dulebohn SC, Di
Napoli R. Features, evaluation and treatment coronavirus (COVID-19). Treasure Island, FL: StatPearls Publishing LLC; 2020.

2. AlJehani YA. Risk factors of periodontal disease: Review of the literature. Int J Dent. 2014;2014:182513.

3. Slots J. Human viruses in periodontitis. Periodontol 2000. 2010;53:89-110.

4. Monje A, Aranda L, Diaz KT, Alarcon MA, Bagramian RA, Wang HL, Catena A. Impact of maintenance therapy for the prevention of peri-implant diseases: A systematic review and meta-analysis. J Dent Res. 2016; 95(4):372-9.

5. Dolic M, Bailer J, Staehle HJ, Eickholz P. Psychosocial factors as risk indicators of periodontitis. J Clin Periodontol. 2005;32(11):1134-40.

6. Flanagan D. Stress related peri-implant bone loss. J Oral Implantol. 2010;36(4):325-7.

7. To KK-W, Tsang OT-Y, Yip CC-Y, Chan K-H, Wu TC, Chan JM-C, Leung W-S, Chik TS-H, Choi CY-C, Kandamby DH, Lung DC, Tam AR, Poon RW-S, Fung AYF, Hung IF-N, Cheng VC-C, Chan JF-W, Yuen K-Y. Consistent detection of 2019 novel coronavirus in saliva. Clin Infect Dis. 2020; ciaa149. doi: 10.1093/cid/ ciaa149.

8. Parhizkar A, Shamszadeh S, Mardani M, Asgary S. Dental considerations after the outbreak of 2019 novel coronavirus disease: A review of literature. Arch Clin Infect Dis. 2020;15(2):e103257.

9. Carreras-Presas CM, Sanchez JA, Lopez-Sanchez AF, Jane-Salas E, Somacarrera Perez ML. Oral vesiculobullous lesions associated with SARS-CoV-2 infection. Oral Dis. 2020. doi: 10.1111/odi.13382.

10. Mombelli A. Maintenance therapy for teeth and implants. Periodontol 2000. 2019;79(1):190-9.

11. Jin YH, Cai L, Cheng ZS, Cheng H, Deng T, Fan YP, Fang C, Huang D, Huang LQ, Huang Q, Han Y. A rapid advice guideline for the diagnosis and treatment of 2019 novel coronavirus (2019-nCoV) infected pneumonia (standard version). Mil Med Res. 2020;7(1):4.

12. Retamal-Valdes B, Soares GM, Stewart B, Figueiredo LC, Faveri M, Miller S, Zhang YP, Feres M. Effectiveness of a pre-procedural mouthwash in reducing bacteria in dental aerosols: Randomized clinical trial. Braz Oral Res. 2017;31:e21.

13. Peng X, Xu X, Li Y, Cheng L, Zhou X, Ren B. Transmission routes of 2019-nCoV and controls in dental practice. Int J Oral Sci. 2020;12(1):9. 